\title{
Comparison of the Efficacy of Tibolone and Transdermal Estrogen in Treating Menopausal Symptoms in Postmenopausal Women
}

\author{
Hyun Kyun Kim, Sung Hye Jeon, Ki-Jin Ryu, Tak Kim, Hyuntae Park \\ Department of Obstetrics and Gynecology, Korea University College of Medicine, Seoul, Korea
}

Objectives: This study aimed to compare the efficacy of tibolone and transdermal estrogen in treating menopausal symptoms in postmenopausal women with an intact uterus.

Methods: Overall, 26 women consumed tibolone orally and 31 women received transdermal estrogen gel mixed with progestogen. The menopause rating scale (MRS) was used to assess their menopausal symptoms at their first outpatient visit and 6 months later.

Results: The transdermal estrogen group showed significant improvements in more items of the MRS questionnaire. There was a favorable change in body weight in the transdermal estrogen group compared with that in the tibolone group. Depressive mood, irritability, physical and mental exhaustion, sexual and bladder problems, and joint and muscular discomfort improved only in the transdermal estrogen group, whereas heart discomfort and vaginal dryness improved only in the tibolone group. Nevertheless, the intergroup differences in each item were insignificant after adjusting for body mass index and hypertension, which differed before treatment.

Conclusions: Both the therapeutic options improved menopausal symptoms within 6 months of use. However, transdermal estrogen appeared to be more effective in preventing weight gain in menopausal women than tibolone.

Key Words: Hormone replacement therapy, Menopause, Tibolone, Transdermal estrogens

\section{INTRODUCTION}

Menopausal symptoms such as hot flushes, affect up to $75 \%$ of menopausal women, with ethnicity-induced variations [1]. For most postmenopausal women, the symptoms last for 3-4 years, but may continue for $\geq$ 10 years in others [2]. Systemic estrogen therapy is the most effective vasomotor symptom treatment and is currently approved by the U.S Food and Drug Administration.

Many studies including randomized controlled trials (RCT) have shown that estrogen monotherapy or estrogen and progestogen co-therapy increase the risk of a thromboembolic event $[3,4]$. Oral hormone re- placement therapy (HRT) increases blood coagulability by increasing clotting factor levels and decreasing antithrombin activity [3]. Therefore, alternative HRTs are needed for postmenopausal women with high risk factors.

Transdermal estrogen is an option absorbed through capillaries in the skin, hence, bypasses the liver and gastrointestinal tract. Compared to oral therapy, it is known to pose a lower risk of thromboembolic events or stroke, owed to its lower influence on Creactive protein, matrix metalloproteinase- 9 , and sex hormone-binding globulin [5]. Thus, it may be safe for postmenopausal women with high risk factors. Additionally, many recent guidelines recommend the use of

Received: May 29, 2019 Revised: October 29, 2019 Accepted: November 7, 2019

Address for Correspondence: Hyuntae Park, Department of Obstetrics and Gynecology, Korea University College of Medicine, 73 Goryeodaero, Seongbuk-gu, Seoul 02841, Korea

Tel: 82-2-920-6824, E-mail: cyberpelvis@gmail.com, ORCID: https://orcid.org/0000-0002-3364-4860 
transdermal HRT in patients with thrombosis or cardiovascular risk factors.

Oral tibolone is widely used in many countries such as Europe and Australasia, but not in America. In the body, it breaks down into three metabolites, which have effects similar to those of both estrogen and progesterone. At the endometrial level, it exerts a tissue-specific progestogenic effect, whereas it has an estrogenic effect on climacteric symptoms and bone [6]. Tibolone is known for its high ability to relieve menopausal and urogenital symptoms and improve bone density, with fewer effects on the endometrium and breasts [7]. In certain cases, due to its ease of administration and induction of less vaginal bleeding and breast tenderness, tibolone is favored over other standard HRTs [8]. It also has positive effects on sexual well-being and mood [7].

Although several studies have compared the effects of tibolone and oral estrogen on menopausal symptoms [9-11], only a few have compared those of tibolone and transdermal estrogen. Thus, this study aimed to compare the effect of tibolone and transdermal estrogen on menopausal symptoms using the menopause rating scale (MRS) questionnaire.

\section{MATERIALS AND METHODS}

This study comprised of a retrospective chart review of Korea University Anam Hospital outpatients, who

Table 1. Demographic characteristics and baseline laboratory tests of the tibolone and transdermal estrogen groups

\begin{tabular}{|c|c|c|c|}
\hline Characteristics & Tibolone & Transdermal estrogen & $P$ value $^{\mathrm{a}}$ \\
\hline Age (yr) & $51.23 \pm 3.20$ & $50.52 \pm 4.16$ & 0.47 \\
\hline Weight (kg) & $54.32 \pm 7.34$ & $58.80 \pm 8.28$ & 0.03 \\
\hline Height (cm) & $158.31 \pm 5.16$ & $157.60 \pm 4.41$ & 0.57 \\
\hline Body mass index $\left(\mathrm{kg} / \mathrm{m}^{2}\right)$ & $21.63 \pm 2.29$ & $23.68 \pm 3.25$ & 0.01 \\
\hline \multicolumn{4}{|l|}{ Comorbidity } \\
\hline Diabetes mellitus & $0(0.0)$ & $4(12.9)$ & 0.11 \\
\hline Hypertension & $1(3.8)$ & $6(19.4)$ & 0.02 \\
\hline Cardiovascular disease & $0(0.0)$ & $0(0.0)$ & \\
\hline Stroke & $0(0.0)$ & $0(0.0)$ & \\
\hline Thyroid & & & 0.85 \\
\hline Normal & $22(84.6)$ & 27 (87.1) & \\
\hline Hypothyroidism & $1(3.8)$ & $2(6.5)$ & \\
\hline Hyperthyroidism & $2(7.7)$ & $1(3.2)$ & \\
\hline Thyroid cancer & $1(3.8)$ & $1(3.2)$ & \\
\hline Parity & & & 0.71 \\
\hline 0 & $3(11.5)$ & $5(16.1)$ & \\
\hline 1 & $2(7.7)$ & $1(3.2)$ & \\
\hline 2 & $20(76.9)$ & $22(71.0)$ & \\
\hline 3 & $1(3.8)$ & $3(9.7)$ & \\
\hline \multicolumn{4}{|l|}{ Baseline tests (score) } \\
\hline Aspartate aminotransferase & $22.19 \pm 5.94$ & $26.05 \pm 16.33$ & 0.32 \\
\hline Alanine aminotransferase & $17.88 \pm 4.80$ & $26.57 \pm 22.82$ & 0.10 \\
\hline Low density lipoprotein cholesterol & $128.91 \pm 31.68$ & $114.80 \pm 40.29$ & 0.34 \\
\hline High density lipoprotein cholesterol & $58.58 \pm 15.63$ & $56.56 \pm 13.93$ & 0.72 \\
\hline Total cholesterol & $205.44 \pm 28.02$ & $202.20 \pm 43.22$ & 0.79 \\
\hline Triglyceride & $101.00 \pm 30.09$ & $157.44 \pm 83.61$ & 0.01 \\
\hline
\end{tabular}

Data are presented as mean \pm standard deviation or number $(\%)$.

${ }^{a}$ Student's $t$ test or Fisher's exact test. 
for the first time commenced HRT for menopausal symptom relief. With approval from the Institutional Review Board, the outpatients were prescribed HRT by the same physician from March 2013 to March 2016 (IRB No. 2019AN0516). Hormone therapy was indicated for women with an intact uterus, who wanted menopausal symptom treatment, and it was contraindicated in those with known or suspected breast or endometrial cancer, undiagnosed abnormal uterine bleeding, or cardiovascular, active liver, or gallbladder disease.

Patient MRSs were determined during their first visit and 6 months later. Postmenopausal status was defined as $\geq 12$ consecutive months of amenorrhea. After providing informed consent, patients chose their HRT. Tibolone $(2.5 \mathrm{mg}$ ) or $1.5 \mathrm{mg}$ transdermal estrogen (Estreva gel; Theramex, London, UK) with daily dydrogesterone (10-20 mg) was prescribed to each group. Twenty-six and 31 postmenopausal Korean women, who met the research criteria and whose records and results were available, were allocated to the tibolone and transdermal estrogen groups, respectively.

To account for menopausal symptom changes, the Korean version of the MRS questionnaire, consisting of 11 items worth $0-4$ points each, was used. Its score range is $0-44$, and it has three symptom domains: somatic, psychological, and urogenital. For statistical analyses, the Student's $t$ test, Fisher's exact test, Mann-Whitney $U$ test, and paired $t$ test were used, and a two-sided $P<$ 0.05 was considered statistically significant. Multivariable analysis was performed by analysis of covariance. In this analysis, body mass index (BMI) and hyperten- sion, which differed in baseline, were corrected and analyzed by analysis of covariance (ANCOVA). All statistical analyses were performed using SAS version 9.4 (SAS Institute, Cary, NC, USA).

\section{RESULTS}

In comparing the baseline characteristics of the tibolone and transdermal estrogen groups, we found that their mean ages were similar (early 50s), and there was a significant difference in the BMI and the presence of hypertension (Table 1). The mean BMI was 21.63 and 23.68 in the tibolone and transdermal estrogen groups, respectively. As opposed to one woman in the tibolone group, 6 in the transdermal group had hypertension. The individuals in the transdermal estrogen group were more obese, had more medical problems, and baseline laboratory tests revealed that their serum triglyceride levels were significantly higher.

When baseline laboratory tests and BMI before and after therapy were compared, the tibolone group showed a decrease in high density lipoprotein cholesterol and an increase in low density lipoprotein cholesterol, triglycerides, and BMI. Conversely, the transdermal estrogen group showed a decrease in triglycerides and BMI. However, only the change in BMI was statistically significant (Table 2). In comparing the baseline MRS scores of both groups, we found more vasomotor symptoms and a higher depression incidence in the transdermal estrogen group (Table 3).

In the tibolone group, hot flushes, sweating, heart discomfort, sleep problems, and vaginal dryness as well as

Table 2. Comparison of the changes in baseline tests and body mass index between the tibolone and transdermal estrogen groups before and after therapy.

\begin{tabular}{lccc}
\hline \multicolumn{1}{c}{ Variable } & \multicolumn{2}{c}{ 2nd-1st mean value } & $P$ Transdermal estrogen \\
\cline { 2 - 3 } & Tibolone & & \\
\hline Baseline tests (score) & & $-4.83 \pm 13.17$ & 0.67 \\
Aspartate aminotransferase & $-3.00 \pm 5.52$ & $-8.33 \pm 21.66$ & 0.50 \\
Alanine aminotransferase & $-3.89 \pm 5.84$ & $-9.14 \pm 34.46$ & 0.51 \\
Low density lipoprotein cholesterol & $19.33 \pm 41.53$ & $-0.33 \pm 10.04$ & 0.12 \\
High density lipoprotein cholesterol & $-12.33 \pm 4.93$ & $-3.45 \pm 53.32$ & 0.82 \\
Total cholesterol & $-12.40 \pm 51.06$ & $-40.27 \pm 91.18$ & 0.23 \\
Triglyceride & $19.25 \pm 23.63$ & $-0.11 \pm 1.43$ & 0.01 \\
Body mass index $\left(\mathrm{kg} / \mathrm{m}^{2}\right)$ & $0.65 \pm 0.88$ & & \\
\hline
\end{tabular}

Data are presented as mean \pm standard deviation.

${ }^{a}$ Student's $t$ test or Mann-Whitney $U$ test. 
Hyun Kyun Kim, et al.

Table 3. Comparison of baseline menopause rating scale scores of the tibolone and transdermal estrogen groups

\begin{tabular}{lccc}
\hline Menopause rating scale questions (score) & Tibolone & Transdermal estrogen & $P$ value $^{\mathrm{a}}$ \\
\hline 1 Hot flashes & $1.42 \pm 1.21$ & $2.10 \pm 1.30$ & 0.04 \\
2 Heart discomfort & $1.42 \pm 1.24$ & $1.55 \pm 1.15$ & 0.69 \\
3 Sleep problems & $1.85 \pm 1.29$ & $2.48 \pm 1.34$ & 0.07 \\
4 Depressive mood & $0.81 \pm 0.90$ & $1.52 \pm 1.21$ & 0.01 \\
5 Irritability & $1.23 \pm 1.14$ & $1.58 \pm 1.39$ & 0.30 \\
6 Anxiety & $1.04 \pm 1.25$ & $1.29 \pm 1.24$ & 0.45 \\
7 Physical and mental exhaustion & $1.81 \pm 1.23$ & $2.10 \pm 1.25$ & 0.38 \\
8 Sexual problems & $2.23 \pm 1.53$ & $2.35 \pm 1.45$ & 0.75 \\
9 Bladder problems & $1.15 \pm 1.22$ & $1.61 \pm 1.41$ & 0.19 \\
10 Dryness of vagina & $1.73 \pm 1.59$ & $1.68 \pm 1.68$ & 0.90 \\
11 Joint discomfort & $2.12 \pm 1.37$ & $2.39 \pm 1.52$ & 0.48 \\
Somato-vegetative symptoms (1,2,3,11) & $6.81 \pm 3.49$ & $8.39 \pm 3.37$ & 0.08 \\
Psychological symptoms (4,5,6,7) & $4.88 \pm 3.53$ & $6.32 \pm 4.11$ & 0.16 \\
Urogenital symptoms (8,9,10) & $5.12 \pm 2.75$ & $5.68 \pm 3.81$ & 0.53 \\
Total & $16.81 \pm 7.26$ & $20.23 \pm 9.29$ & 0.13 \\
\hline
\end{tabular}

Data are presented as mean \pm standard deviation.

a'Student's $t$ test.

Table 4. Comparison of the 1st and 2nd menopause rating scale (MRS) scores of the tibolone and transdermal estrogen groups

\begin{tabular}{|c|c|c|c|c|c|c|}
\hline \multirow{2}{*}{ MRS questions } & \multicolumn{3}{|c|}{ Tibolone } & \multicolumn{3}{|c|}{ Transdermal estrogen } \\
\hline & 1st MRS & 2nd MRS & $P$ value $^{\mathrm{a}}$ & 1st MRS & 2nd MRS & $P$ value $^{\mathrm{a}}$ \\
\hline 1 Hot flashes & $1.42 \pm 1.21$ & $0.88 \pm 0.91$ & 0.019 & $2.10 \pm 1.30$ & $1.10 \pm 1.08$ & $<0.001$ \\
\hline 2 Heart discomfort & $1.42 \pm 1.24$ & $0.85 \pm 0.83$ & 0.029 & $1.55 \pm 1.15$ & $1.10 \pm 0.98$ & 0.050 \\
\hline 3 Sleep problems & $1.85 \pm 1.29$ & $1.35 \pm 1.35$ & 0.029 & $2.48 \pm 1.34$ & $1.48 \pm 1.31$ & $<0.001$ \\
\hline 4 Depressive mood & $0.81 \pm 0.90$ & $0.50 \pm 0.58$ & 0.088 & $1.52 \pm 1.21$ & $1.03 \pm 1.17$ & 0.033 \\
\hline 5 Irritability & $1.23 \pm 1.14$ & $0.73 \pm 0.92$ & 0.050 & $1.58 \pm 1.39$ & $1.00 \pm 1.29$ & 0.001 \\
\hline 6 Anxiety & $1.04 \pm 1.25$ & $0.77 \pm 0.91$ & 0.250 & $1.29 \pm 1.24$ & $0.97 \pm 1.14$ & 0.076 \\
\hline 7 Physical and mental exhaustion & $1.81 \pm 1.23$ & $1.62 \pm 1.02$ & 0.370 & $2.10 \pm 1.25$ & $1.55 \pm 1.18$ & 0.005 \\
\hline 8 Sexual problems & $2.23 \pm 1.53$ & $1.73 \pm 1.28$ & 0.100 & $2.35 \pm 1.45$ & $1.74 \pm 1.48$ & 0.034 \\
\hline 9 Bladder problems & $1.15 \pm 1.22$ & $1.04 \pm 1.00$ & 0.690 & $1.61 \pm 1.41$ & $0.97 \pm 1.08$ & 0.002 \\
\hline 10 Dryness of vagina & $1.73 \pm 1.59$ & $0.96 \pm 1.15$ & 0.038 & $1.68 \pm 1.68$ & $1.16 \pm 1.51$ & 0.088 \\
\hline 11 Joint discomfort & $2.12 \pm 1.37$ & $1.58 \pm 1.24$ & 0.079 & $2.39 \pm 1.52$ & $1.74 \pm 1.48$ & 0.039 \\
\hline Somato-vegetative symptoms $(1,2,3,11)$ & $6.81 \pm 3.49$ & $4.69 \pm 2.84$ & 0.001 & $8.39 \pm 3.37$ & $5.45 \pm 3.60$ & $<0.001$ \\
\hline Psychological symptoms $(4,5,6,7)$ & $4.88 \pm 3.53$ & $3.62 \pm 2.76$ & 0.065 & $6.32 \pm 4.11$ & $4.35 \pm 3.96$ & 0.001 \\
\hline Urogenital symptoms $(8,9,10)$ & $5.12 \pm 2.75$ & $3.73 \pm 2.62$ & 0.049 & $5.68 \pm 3.81$ & $4.03 \pm 3.29$ & 0.019 \\
\hline Total MRS score & $16.81 \pm 7.26$ & $12.04 \pm 6.32$ & 0.003 & $20.23 \pm 9.29$ & $13.77 \pm 9.34$ & $<0.001$ \\
\hline
\end{tabular}

Data are presented as mean \pm standard deviation.

${ }^{a}$ Paired $t$ test. 
Table 5. Comparison of the difference between the 2nd and 1st menopause rating scale (MRS) scores (2nd-1st) of the tibolone and transdermal estrogen groups

\begin{tabular}{lcccc}
\hline \multicolumn{1}{c}{ MRS questions } & \multicolumn{2}{c}{ 2nd-1st MRS } & $\begin{array}{c}\text { Mean difference } \\
\text { (Tibolone-transdermal } \\
\text { estrogen MRS) }\end{array}$ & $P$ value \\
\cline { 2 - 4 } 1 Hot flashes & Tibolone & Transdermal estrogen & 0.55 & 0.27 \\
2 Heart discomfort & -0.46 & -1.00 & 0.10 & 0.78 \\
3 Sleep problems & -0.47 & -0.57 & 0.58 & 0.26 \\
4 Depressive mood & -0.54 & -1.12 & 0.23 & 0.64 \\
5 Irritability & -0.22 & -0.46 & 0.14 & 0.78 \\
6 Anxiety & -0.47 & -0.61 & 0.03 & 0.98 \\
7 Physical and mental exhaustion & -0.39 & -0.41 & 0.55 & 0.08 \\
8 Sexual problems & 0.00 & -0.55 & 0.55 & 0.20 \\
9 Bladder problems & 0.11 & -0.44 & 0.58 & 0.38 \\
10 Dryness of vagina & -0.16 & -0.74 & 0.09 & 0.84 \\
11 Joint discomfort & -0.55 & -0.64 & 0.33 & 0.66 \\
Somato-vegetative symptoms (1,2,3,11) & -1.07 & -0.26 & 1.42 & 0.30 \\
Psychological symptoms (4,5,6,7) & -1.06 & -2.89 & -2.04 & 0.98 \\
Urogenital symptoms (8,9,10) & -0.70 & -1.81 & -6.68 & 3.39 \\
Total MRS score & -3.29 & & & 0.43 \\
\hline
\end{tabular}

${ }^{\mathrm{a}}$ Analysis of covariance (ANCOVA).

somato-vegetative and urogenital symptoms, measured by the MRS score, were significantly improved (Table 4). The MRS score of other items was also decreased, although not significantly. In the transdermal estrogen group, there were significant improvements in hot flushes, sweating, sleep problems, depressive mood, irritability, physical and mental exhaustion, sexual problems, bladder problems, and joint and muscular discomfort. Significant improvements in somato-vegetative, psychological, and urogenital symptoms were also observed.

Finally, we determined if significant differences existed between the two groups. After adjusting for BMI and hypertension, which were different at baseline, the difference in the improvement of menopausal symptoms between the two groups was not statistically significant (Table 5).

\section{DISCUSSION}

Few studies have compared the effectiveness of tibolone and estrogen for the relief of menopausal symptoms. One RCT comparing the effects of $2.5 \mathrm{mg}$ tibolone and $1.0 \mathrm{mg} 17 \beta$-estradiol (E2)/0.5 mg nor- ethisterone acetate (NETA) on vasomotor symptoms, found that there was similar improvement in both groups within the first 3 months. The frequency of vaginal bleeding and breast tenderness were reportedly significantly lower in the tibolone group than in the E2/ NETA group [9]. With regard to the vasomotor symptom-relieving ability of tibolone and estrogen/progestogen, two recent meta-analyses reported no significant differences [10], whereas one concluded that tibolone was less effective [11]. However, considering the differences in the hormone formula and the questionnaire used for vasomotor symptom analysis, their data were insufficient for a conclusive meta-analysis.

To our knowledge, the effects of tibolone and transdermal estrogen on menopausal symptoms in surgically menopaused women have only been compared in one study [12]. The results showed that, in these women, tibolone was more effective at improving health-related quality of life, possibly because its androgenic effect lowers sex hormone-binding globulin levels, and results in improvements in pituitary $\beta$-endorphins-induced sexual problems or mood. This study was conducted on surgical menopausal women, but we included nature menopausal women in our study. This difference 
in the subject group may have affected the outcome.

To the best of our knowledge, the present study is the first to compare the effectiveness of tibolone and transdermal estrogen on menopausal symptoms in naturally menopaused women. In this study, the transdermal estrogen group showed statistically significant improvements in a higher number of the MRS questionnaire items. Depressive mood, irritability, physical and mental exhaustion, sexual and bladder problems, and joint and muscular discomfort improved only in the transdermal estrogen group.

Several possible explanations can be given. Firstly, transdermal estrogen is a more effective reliever of menopausal symptoms in naturally menopaused women than tibolone. Estradiol gel produces relatively stable serum estradiol levels, and therapeutic estradiol levels similar to those seen with other formulations, routes of administration, and dosages. The transdermal route allows direct delivery to the systemic circulation, avoiding gastrointestinal and first-pass hepatic metabolism and elimination. In contrast, oral medication causes fluctuations in serum levels due to absorption and metabolism [13]. Secondly, the co-administered dydrogesterone enhanced the menopausal symptomrelieving effect of transdermal estrogen. Lastly, baseline characteristics such as BMI, hypertension, and serum triglyceride levels were different between the groups, and menopausal symptoms in the transdermal estrogen group at the start of the therapy were worse than those in the tibolone group (the first avereage MRS scores were 20.23 and 16.81 in the transdermal estrogen and tibolone groups, respectively). Overweight women reportedly have more severe vasomotor symptoms than women with normal weight [14]. Generally, HRTs may be more efficacious in severe menopausal cases. After adjusting for BMI and hypertension, which were different at baseline, the intergroup differences after therapy were not statistically significant.

A limitation of this study is the small number of samples. Additionally, the limitations of this retrospective study make comparing these drugs difficult. Furthermore, bias might have been introduced during the selection process, by choosing higher risk subjects such as obese, hypertensive, and diabetic patients, for the transdermal estrogen group. The study, however, provides useful information on both drugs by comparing pre- and post-therapy characteristics in both groups.

It is noteworthy that transdermal estrogen had a favorable effect on body weight in this study. A possible drug-related adverse effect of tibolone includes weight gain [9]. Estrogen replacement therapy is known to be unrelated to weight gain in comparison with a placebo when taken in the oral form [15]. Thus, the observed positive effect of transdermal estrogen on body weight needs further investigation.

In our study, there was no significant difference in the treatment effect of menopausal symptom between two groups when BMI and hypertension were adjusted. However, as shown in the results, transdermal estrogen showed statistically significant effect in 6 items, and tibolone was effective in two items. Therefore, transdermal estrogen can be one of the options to address the health and quality of life problems of postmenopausal women. Menopausal women should be aware of the various treatment options, and to meet their preferences, more randomized and prospective studies should be performed in larger scale in order to compare the efficacy of transdermal estrogen and tibolone.

\section{ACKNOWLEDGMENTS}

This study was supported by the National Research Foundation of Korea (NRF) (grant number: 2013R1A1A1010044).

\section{CONFLICT OF INTEREST}

No potential conflict of interest relevant to this article was reported.

\section{REFERENCES}

1. Shifren JL, Schiff I. Menopause. In: Berek JS, Novak E, editors. Berek \& Novak's gynecology. 15th ed. Philadelphia (PA): Lippincott Williams \& Wilkins; 2012. pp.1233-48.

2. Schiff I, Regestein Q, Tulchinsky D, Ryan KJ. Effects of estrogens on sleep and psychological state of hypogonadal women. JAMA 1979; 242: 2405-7.

3. Anderson GL, Limacher M, Assaf AR, Bassford T, Beresford SA, Black $\mathrm{H}$, et al. Effects of conjugated equine estrogen in postmenopausal women with hysterectomy: the Women's Health Initiative randomized controlled trial. JAMA 2004; 291: 1701-12.

4. Hulley SB, Grady D. The WHI estrogen-alone trial--do things look any better? JAMA 2004; 291: 1769-71.

5. Goodman MP. Are all estrogens created equal? A review of oral vs. transdermal therapy. J Womens Health (Larchmt) 2012; 21: 161-9.

6. Fedele L, Bianchi S, Raffaelli R, Zanconato G. Comparison of transdermal estradiol and tibolone for the treatment of oopho- 
rectomized women with deep residual endometriosis. Maturitas 1999; 32: 189-93.

7. Kenemans P, Speroff L; International Tibolone Consensus Group. Tibolone: clinical recommendations and practical guidelines. A report of the International Tibolone Consensus Group. Maturitas 2005; 51: 21-8.

8. Ross LA, Alder EM. Tibolone and climacteric symptoms. Maturitas 1995; 21: 127-36.

9. Hammar ML, van de Weijer P, Franke HR, Pornel B, von Mauw EM, Nijland EA. Tibolone and low-dose continuous combined hormone treatment: vaginal bleeding pattern, efficacy and tolerability. BJOG 2007; 114: 1522-9.

10. Burbos N, Morris EP. Menopausal symptoms. BMJ Clin Evid 2011; 2011: 0804.

11. Formoso G, Perrone E, Maltoni S, Balduzzi S, D'Amico R, Bassi
C, et al. Short and long term effects of tibolone in postmenopausal women. Cochrane Database Syst Rev 2012; (2): CD008536.

12. Bhattacharya SM, Jha A. Effects of transdermal estradiol gel and oral tibolone on health-related quality of life after surgical menopause. Int J Gynaecol Obstet 2010; 110: 213-6.

13. Archer DF. Estradiol gel: a new option in hormone replacement therapy. OBG Manag 2004; 16: 46-66.

14. Ryu KJ, Park HT, Kwon DH, Yang KS, Kim YJ, Yi KW, et al. Vasomotor symptoms and metabolic syndrome in Korean postmenopausal women. Menopause 2015; 22: 1239-45.

15. Norman RJ, Flight IH, Rees MC. Oestrogen and progestogen hormone replacement therapy for peri-menopausal and postmenopausal women: weight and body fat distribution. Cochrane Database Syst Rev 2000; (2): CD001018. 